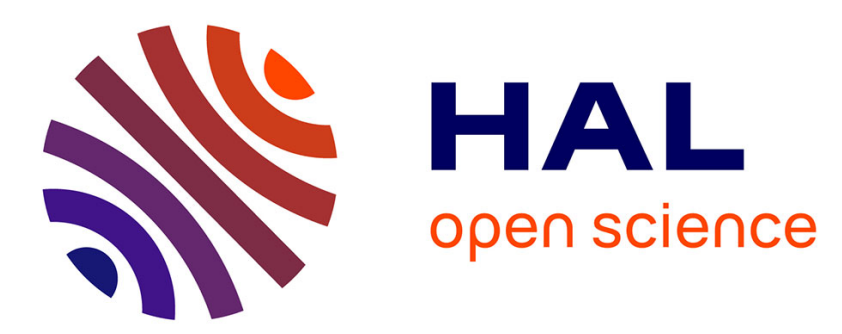

\title{
Ranges of the least uncomfortable joint angles for assessing automotive driving posture
}

\author{
Junfeng Peng, Xuguang Wang, Lisa Denninger
}

\section{To cite this version:}

Junfeng Peng, Xuguang Wang, Lisa Denninger. Ranges of the least uncomfortable joint angles for assessing automotive driving posture. Applied Ergonomics, 2017, 61, pp.12-21. 10.1016/j.apergo.2016.12.021 . hal-01737314

\section{HAL Id: hal-01737314 \\ https://hal.science/hal-01737314}

Submitted on 19 Mar 2018

HAL is a multi-disciplinary open access archive for the deposit and dissemination of scientific research documents, whether they are published or not. The documents may come from teaching and research institutions in France or abroad, or from public or private research centers.
L'archive ouverte pluridisciplinaire HAL, est destinée au dépôt et à la diffusion de documents scientifiques de niveau recherche, publiés ou non, émanant des établissements d'enseignement et de recherche français ou étrangers, des laboratoires publics ou privés. 


\section{Ranges of the least uncomfortable joint angles for assessing automotive driving posture}

Junfeng Peng a,b,c,d, Xuguang Wang ${ }^{\mathrm{a}, \mathrm{b}, \mathrm{c}^{*}}$, Lisa Denninger ${ }^{\mathrm{d}}$

${ }^{a}$ Université de Lyon, F-69622, Lyon, France

${ }^{b}$ Université Claude Bernard Lyon 1, Villeurbanne

'IFSTTAR, UMR_T9406, LBMC Laboratoire de Biomécanique et mécanique des chocs, F69675,Bron;

${ }^{d}$ PSA Peugeot-Citroën, Sochaux

* Corresponding author: Xuguang Wang, LBMC-Ifsttar, 25 av. F. Mitterrand, case 24, 69675 BRON Cedex, France

Tel: $+33(0) 4.72 .14 .24 .51$

Fax: $+33(0) 4.72 .14 .23 .60$

Email: xuguang.wang@ifsttar.fr 


\section{Abstract}

Few investigations have been performed on how the ranges of preferred angles should be used for vehicle interior discomfort evaluation. This study investigated the ranges of the least uncomfortable joint angles considering both inter-individual and intra-individual variability. The driving postures of sixty-one subjects were collected using two multi-adjustable vehicle mock-ups under four test conditions by gradually adding the number of control parameters (constraints), from the "least-constrained" driving condition to the configurations close to currently existing vehicles. With help of subjective discomfort evaluation, the intra-and interindividual variation ranges of least uncomfortable postural angles were quantified. Results show that intra-individual variation ranges of postural angles were much smaller than those of inter-individual variation as expected. An individual may not feel comfortable throughout the whole range of comfortable angles from all participants. Possible relationships between perceived discomfort and ranges of inter and inter individual variations in least uncomfortable angles were explored, suggesting that the inter ranges could be used to detect potential problems of postural discomfort and the intra ranges could be considered as optimum ranges. A three color model, based on the intra-and inter-individual variability ranges of comfortable driving postures, was proposed for ergonomics assessment of a vehicle configuration.

\section{Keywords}

Driving posture; discomfort; digital human modeling; vehicle packaging 


\section{Introduction}

In the expanding global competitive automotive market, vehicles are required to be designed and manufactured in a short term and also to provide a high level of comfort for the target population of more than thousands or even millions of people. To meet these challenges, Digital Human Models (DHMs) are being more and more used for proactive as well as retrospective ergonomics analysis in the automotive vehicle interior design process. With help of a DHM tool, preferred comfortable driving postures can be used for optimizing the vehicle interior layout including the adjustment range of interior components, such as seat, steering wheel at the early phase of design. For example, Vogt et al. (2005) tested four seating concepts for vehicle interior design with help of the RAMSIS digital human modeling tool. For this, a comfortable driving posture regardless of driver's anthropometry was used. Comfortable driving postures can also be used to assess different design propositions in a later phase of vehicle design. Actually, the ergonomics requirements may not be $100 \%$ satisfied because of other considerations such as styling, legal requirements, aerodynamics, and safety. It means that the compromised design may not accommodate all target drivers. Postural discomfort could be assessed by comparing the ranges of comfortable joint angles with the posture adopted by a driver, as implemented in RAMSIS (Meulen and Seidl 2007).

In the past, a few studies recommended the range of 'comfortable' or 'preferred' joint angles for automotive driving. Rebiffé (1969) analyzed the main driving tasks and suggested the ranges of comfortable joint angles in the sagittal plane. It should be noted that, Rebiffé's recommendation was based on the author's experience without experimental evidence. Some other researches (Seidle 1994, Porter and Gyi 1998, Park et al. 2000, Hanson et al. 2006) experimentally investigated the preferred driving posture using a multi-adjustable vehicle mockup with extended range of adjustments by assuming that preferred driving posture would also be comfortable. Kyung et al. (2009) questioned whether the preferred posture is necessarily a comfortable one. They suggested considering subjective discomfort and comfort ratings for selecting comfortable postures to determine the comfortable joint angle ranges. In the past, the comfortable joint angle intervals were obtained from all participants, representing rather interindividual than intra-individual variation. As intra-individual postural variation is much smaller than inter-individual variation in general, a driver may not feel comfortable throughout the whole inter-individual range of comfortable postures from all participants. Thus, it would also be interesting to define the ranges of comfortable angles based on intra-individual variation. Moreover, few investigations have been performed to see whether the range of preferred angles 
could be used for discomfort evaluation. Could we assume that a vehicle configuration allowing all joint angles of a driver inside the suggested intervals is comfortable for driving? If one or several angles are outside the comfortable intervals, how does the discomfort change? These were rarely discussed in the literature. As argued by Zhang and Helander (1996) and more recently by Vink and Hallbeck (2012), discomfort and comfort are two distinct concepts. Discomfort is related to physical characteristics of the environment like posture, stiffness and fatigue, whereas comfort is related to luxury, relaxation or being refreshed. In the present paper, postural discomfort was investigated.

The main goal of this study was therefore to investigate the range of comfortable joint angles considering both inter-individual and intra-individual variability for automotive driving postural discomfort evaluation. For this, data were collected from less-constrained vehicle configurations with low discomfort, more constrained ones close to real vehicles as well as unrealistic ones with high discomfort. Comfortable joint angles and their range of variation were first obtained from the configurations with low discomfort. Then a three color model, based on the intra-and inter-individual variability ranges of comfortable driving postures, was proposed and tested using data from more constrained test conditions.

\section{Materials and methods}

\section{Experimental data}

The data used in the present study were collected from 61 participants using two multiadjustable vehicle mockups, as shown in Figure 1 and Figure 2. One, named 'Mockup 1 (M1)', could be adjusted easily by subjects themselves through an intuitive touch-screen, while the other, named 'Mockup 2 (M2)', had the adjustments mainly controlled by a computer and could easily load a predefined vehicle configuration. A same Peugeot 508 seat, considered as a highend product with electric adjustments in fore-aft position, height, lumbar support, cushion angle and back inclination, was used for both mockups. Main characteristics of the two mockups are summarized in Table 1.

Sixty one drivers, recruited mainly based on stature and sitting height, included both French and Chinese drivers who lived in France. They all had a driving license for more than one year and drove regularly. They did not suffer any neurological or musculoskeletal disorders. The main characteristics of the participants are described in Table 2. The whole experiment was divided into four test conditions by gradually adding the number of control 
parameters (constraints), from the "least-constrained" condition to the configurations close to currently existing vehicles, as shown in Table 3. Only the fore-aft position of the accelerator pedal was controlled in Condition 1, meaning that participants were allowed to use all adjustments available as long as they respected the instruction for "a natural driving". In Condition 2, seat height was added as control variable and three levels were tested. In Condition 3, one more vehicle parameters (steering wheel fore-aft position, clutch pedal foreaft position, seat cushion angle) was controlled in addition to the seat height fixed at $300 \mathrm{~mm}$. Condition 4 simulated 27 different configurations close to currently existing vehicles. Only fore-aft seat position and seat back angle were allowed to be adjusted.

Figure 1.

Figure 2.

Table 1.

Table 2.

Table 3.

All 61 subjects participated in the "less-constrained" experiments (C1, C2 and C3) using the mockup 1 and only 55 of them participated in the "more-constrained" experiment (C4) using the mockup 2. For each test configuration, subjects were asked to find their preferred vehicle parameters by using all available adjustments according to experimental condition. The initial positions of available adjustments were set randomly to one of the two extremes such that participants had to use the available adjustments allowed by test condition. Once the subjects found their preferred driving positions, a full movement of depressing clutch pedal was recorded by a Vicon motion caption system. Reflective markers were attached to the body as well as to all moveable parts of the mock-up. The participants were instructed to place their hands on the steering wheel at the 10-to-2 o'clock position and to look forward as they were driving. They were asked to put their left foot on the foot rest, to fully depress the clutch pedal to its end, and then to move the left foot back on the foot rest. The right foot was asked to put on the accelerator without depressing. Subjects were required to leave the mockup after each trial and the adjustments were reset for a new test condition. Trial order was randomized within each test session while the four sessions were performed in order.

For each test configuration, after having found the preferred posture and before getting out of the seat for another test condition, participants were asked to verbally rate the configuration using a modified CP-50 category partition scale CP50 scale (Chevalot and Wang, 2004, see 
also Shen and Parson, 1997 for a comparative study on rating scales), as shown in Figure 3. The scale was put in front of the subject and was visible all the time. They were instructed to first select a category among seven responses (imperceptible, very low, low, medium, high, very high, extremely high), then to refine their judgement by choosing a number from 1 to 10 within the selected category. The real scale from 0 to 50 and more (original CP-50) was hidden from the subject in order to give priority to the category choice and not to a numerical value.

Figure 3.

In total, a maximum number of 41 trials were asked to perform for each participant. The whole experiment took about 90-120 minutes for the experiment with Mockup 1 and 60-90 minutes for the experiment with Mockup 2.

The whole clutch pedal depressing movement was reconstructed using the RAMSIS human model by minimizing the distance between the captured and model-based markers positions (see Ausejo and Wang 2008, for the principle of motion reconstruction). Three instants of a clutching movement were identified to describe the driving postures at the rest (Re), beginning (De) and end of clutch pedal depression (Fi). A set of postural parameters of each instant, defined in

Table 4 and illustrated in Figure 4, were calculated from reconstructed joint centers. If not indicated explicitly, the angles at the rest posture are presented.

Figure 4.

Table 4

The time spent on each trial was largely dependent on the number of available adjustments and on participant, varying from 2 minutes for Condition 4 with only two adjustments (fore-aft seat position and seat back angle) to 15 minutes for Condition 1 with 16 available adjustments. Not all planned trials were finished in time. Seven and thirteen trials under Condition 2 and Condition 3 were missed. Five trials under Condition 4 were discarded due to the problem of motion capture.

\section{Data analysis}

In order to determine range of least uncomfortable angles, the distribution of each angle was examined for the data from each participant and also from all participants together. The $5^{\text {th }}$, median $\left(50^{\text {th }}\right)$ and $95^{\text {th }}$ percentiles were estimated. Contingency tables relating discomfort categorical responses and angles were also generated. Distributions of the categorical responses 
under different conditions were tested by a Chi squared test. Statistical significance level was fixed at $\mathrm{p}=0.05$.

A test configuration was repeated three times under Condition 2 and Condition 4, allowing an assessment of the intra-individual variability of discomfort rating. The intra-individual variability was calculated as the maximum difference in rating between three repetitions.

\section{Results}

\section{Discomfort ratings}

Under Condition 2, the maximum difference in rating between three repetitions (intraindividual variability) was smaller than 10 , whereas 15 participants had a maximum difference greater than 10 under Condition 4, implying that the rating of a same test configuration changed the category from trial to trial. This also probably suggests difficulty in using the rating scale for these volunteers.

In order to see whether subjective rating changed with test conditions, the percentages of different discomfort categories of the 4 experimental conditions are presented in Table 5. For the least-constrained condition (Condition 1), all participants rated it with a discomfort score less than 10 ('imperceptible' or 'very slight'), while its percentage decreased to $23.6 \%$ for Condition 4. For the category "average" with $20<$ Rating $\leq 30$, its percentage increased consistently from $0 \%$ in Condition 1 to $30.1 \%$ in Condition 4 . As expected, the percentage of configurations with higher discomfort rating increased significantly from Condition 1 to Condition 4 . It should be noted that the number of control parameters (constraints) was increased gradually from Condition 1 to Condition 4 showing that a more constrained vehicle configuration induced a higher discomfort rating as expected.

Table 5

\section{Correlation between postural angles}

Table 6 presents the coefficients of correlation between main postural angles at the rest from the data of all test conditions. As expected, the postural angles of the right side were highly correlated with those on the left. Strong correlations were also observed between trunk inclination and trunk-thigh angles ( 0.646 for the right side, and 0.639 for the left side), between shoulder and elbow angles ( 0.615 for the right side and 0.668 for the left side), and between 
trunk inclination and shoulder angles ( -0.443 for the right side and -0.47 for the left side). The trunk inclination, knee and elbow angles were little correlated.

Table 6

\section{Intra and inter individual variations of the least uncomfortable postural angles}

All trials corresponding to the discomfort category 'very slight' (with a discomfort rating smaller than 11) regardless of test condition ( 889 over 2302 trials) were retained for calculating the ranges of intra and inter participant variation of the least uncomfortable postures. The median of each postural angle was calculated for each participant at first. Then the distribution of individual medians from all participants was calculated, representing inter individual variability. For intra individual variability, the values of each angle for one subject was firstly centered at his/her individual median, then the distribution of centered values from all participants was calculated. Table 7 gives the $5^{\text {th }}, 50^{\text {th }}, 95^{\text {th }}$ percentiles of individual medians, as well as the $5^{\text {th }}$ and $95^{\text {th }}$ percentiles of the angles centered at corresponding each participant's median. The inter individual range of variation was defined as the difference from the $5^{\text {th }}$ to $95^{\text {th }}$ percentiles of the individual medians among all participants. For the intra individual range of variation, its amplitude was defined from the $5^{\text {th }}$ and $95^{\text {th }}$ percentiles of the centered angles. The inter and intra ranges of variation are compared in Figure 5, clearly showing intra individual variability was much smaller than inter individual variability for all postural angles as expected. For example, trunk inclination varied from $5^{\text {th }}$ to $95^{\text {th }}$ percentiles only about $\pm 5^{\circ}$ around its median individually, while its variation range among different subjects was more than two times $\left(20.8^{\circ}\right)$, from $11.7^{\circ}$ to $32.5^{\circ}$. Big difference in intra and inter variabilities was also observed between different angles. Trunk inclination was the least varied postural angle while the elbow had the highest variability.

In order to see if there are any effects of stature, the ranges of intra individual variation from $5^{\text {th }}$ to $95^{\text {th }}$ percentiles by stature group are listed in Table 8 . for the main postural parameters as well the means and standard deviations of the corresponding individual medians. No significant difference in the individual medians of these postural angles was found between the three stature groups. However different ranges of intra individual variation were observed between the small and tall groups, especially for the elbow and knee angles at the end of clutch pedal 
travel. Smaller range of variation in the knee was observed for short participants $\left(13.6^{\circ}\right.$ versus $\left.19.8^{\circ}\right)$, whereas they had much larger variation in the elbow $\left(36.5^{\circ}\right.$ versus $\left.26.7^{\circ}\right)$.

Table 7

Table 8.

Figure 5.

\section{Boundaries of inter and intra individual range of variation and their relationships with perceived discomfort}

Figure 5 confirms that the inter individual range of variation of the least uncomfortable angles is much larger than that of intra individual variation. As stated in Introduction, a driver may not feel comfortable throughout the whole inter-individual range of the least uncomfortable postures. Thus, it would also be interesting to define their ranges based on intra-individual variation for assessing a vehicle configuration. For the inter individual range of variation of the least uncomfortable angles, called 'inter range', it is proposed as being bounded from the $5^{\text {th }}$ to $95^{\text {th }}$ percentiles of the individual medians for each angle. As the intra individual range of variation, called 'intra range', was estimated from the values centered at each participant's individual median, the median of the distribution of all individual medians was used to define its boundaries for all participants. Boundaries of both inter and intra individual ranges of variation are also listed in Table 7.

As an exploratory attempt for verifying if these intra and inter ranges of the least uncomfortable angles can be used to assess a vehicle configuration, the postural angles of the trials under Condition 4 (C4) close to the dimensions of existing vehicles, were compared with the corresponding intra and inter ranges of the least uncomfortable angles. Trunk inclination, left elbow angle and left knee angle at the end of clutch pedal travel were first selected for the following considerations:

- Trunk inclination, knee and elbow angles were among the least correlated variables at the rest

- The angles of the left and right sides were almost symmetric for the posture at the rest

- Clutch pedal depression mainly modified the angles of the left side

The C4 trials were first divided into two groups, one with all the three key postural angles being within their respective inter or intra range of the least discomfort angles ('inside'), the other with at least one angle being outside the corresponding inter or intra range ('outside'). One 
could expect a higher percentage of the trials with a low discomfort rating ('very slight' or 'slight') in the 'inside' group, while a higher percentage of the trials with high discomfort rating ('medium', 'strong' and 'very strong' discomfort) should be higher in the 'outside' group. This hypothesis is verified in Table 9 by using inter or intra range of the least discomfort angles. When the inter range was used, there were 867 'inside' trials among which $66.2 \%$ had a rating smaller than 20; and 290 'outside" trials among which 59.3\% had a rating greater than 20 . When the intra range was used, only 295 'inside' trials were found among which $74.6 \%$ had a rating smaller than 20. Among 862 'outside' trials, only $45.2 \%$ had a rating greater than 20 , showing that the intra range may be too restrictive. Results suggest that the inter range might be used for detecting uncomfortable posture, whereas the intra range might be appropriate for specifying the least uncomfortable posture.

Table 9

\section{Discussion}

In the present study, the intra and inter individual variations of the least uncomfortable postural angles at the rest, beginning and end of clutch depression were investigated. Two ranges of variation of these angles were proposed, called inter and intra ranges, according to their inter and intra individual variability. As expected, the range of inter individual variation was higher than intra individual range. It was found that inter ranges could be used to detect badly rated postures and the intra ranges could be considered as optimum ranges. In what follows, we'll compare the intra and inter ranges of least uncomfortable joint angles found in the present work with some previous investigations, then propose a driving postural assessment model.

\section{Comparing ranges of least uncomfortable joint angles with other studies}

In most of the earlier studies, only driving posture at the rest was investigated in the sagittal plane. In the present work, a whole left foot clutching movement was recorded from the rest position to the end of full clutch depression. Postural parameters of the whole body at the rest, beginning and end of clutch depression were defined from 3D reconstructed motions with help of a three-dimensional human model. As stated recently by Schmidt et al (2013) in an extensive review on preferred joint angles in automotive sitting posture, comparison of preferred angles 
between different studies is problematic due to differences in the methods used for estimating joint angles and also in the experimental conditions (e.g. instruction given to participants to place the hands on the steering wheel, number of available adjustments, etc). Being aware of this difficulty in comparing the ranges of comfortable angles with other studies, Table 10 compares the inter and intra ranges proposed in the present study with the ranges of preferred/comfortable trunk, trunk-thigh, knee and elbow angles recommended by some earlier studies. Only the studies on comfortable angles for automotive driving posture were selected, as preferred sitting posture is activity dependent. For instance, Kilincsoy et al (2014) and Hiemstra-Van Mastrigt (2015) investigated car and train passenger postures by activity. Kilincsoy et al showed that relaxed postures preferred by car passenger were quite different from those recommended by RAMSIS for driving. For the purpose of comparison, joint angles in the current study were converted into the angles defined in the sagittal plane. For trunk inclination, the proposed intra range $\left(17^{\circ}-27^{\circ}\right)$ is quite close to that recommended by Rebiffé (1969). Kyung (2009) observed a quite large variation from $18^{\circ}$ to $43^{\circ}$; whereas the proposed inter range varies from $12^{\circ}$ to $33^{\circ}$. Note that RAMSIS recommends an optimum trunk inclination of $27^{\circ}$. Concerning trunk-thigh angle, the inter range observed in the present study $\left(82^{\circ}-102^{\circ}\right)$ is between the range obtained by Hanson et al (2006) and that by Park et al (2000). Hanson et al obtained a range from $68^{\circ}$ to $99^{\circ}$ with the mid value much smaller than the present study. This may be due to the difference in the range of seat height and due to subject selection. In Hanson's study, seat height (H30) varied from -20 to $265 \mathrm{~mm}$ while it varied from 250 to $350 \mathrm{~mm}$ in the present study (except Condition 1). Our results showed that trunk-thigh angle increased with seat height (Peng et al, 2015). Besides, our participants were composed of French and Chinese drivers. Results showed that Chinese drivers had a more open trunk-thigh angle than French drivers (Peng et. 2015). For the right knee angle, the inter range by the present study is within the ranges proposed by Rebiffé (1969), Porter and Gyi (1998) and also by Kyung (2008), but quite different from that proposed by Park et al (1999). The right knee angle range by Hanson et al is much larger with its mid value much higher than the present study. For the elbow angle, the proposed inter range is within the ranges proposed by most of earlier studies.

From an extensive review on sitting biomechanics for optimizing automotive seat design, Harrison et al. (2000) suggested that seat backrest (trunk) angle to the vertical should not be exceeded $30^{\circ}$ for preventing from large head flexion angle, though a backrest angle of $30^{\circ}$ was reported as optimum for low inter disc pressure and muscle activities. It could be reduced to $10^{\circ}$ with seat bottom incline to $5^{\circ}$ and arm rests added while maintaining low muscle activities 
and low disc pressure. Our observations for both inter and intra range of comfortable angles agree with their suggestion. However, for the trunk-thigh angle, our findings for the right side were smaller than $105^{\circ}$, an angle suggested as necessary to preserve lumbar lordosis.

Table 10

\section{Proposition of a three-color model for assessing a vehicle configuration}

Despite the difficulty in using CP-50 scale for some participants with a poor repeatability of subjective rating, results suggest that subjective rating with CP-50 scale was able to reflect different discomfort level when changing vehicle configuration (Table 5). Inter and intra ranges of comfortable angles, as defined in Table 7, were also used to look at the distribution of discomfort categorical responses in function of whether an angle is inside or outside its intra and inter ranges of the least discomfort. Trunk inclination, left elbow angle and left knee angle at the end of clutch depression were proposed as main postural variables for assessing global discomfort of a vehicle configuration. Nearly $60 \%$ of the trials with at least one angle being outside its inter range had a discomfort rating higher than 20 , whereas more than $70 \%$ of the trials with all three angles within their intra ranges had a score smaller than 20 (Table 9). This suggests that the inter ranges could be used to detect potential problems of postural discomfort of a vehicle and the intra ranges could be considered as optimum ranges. Therefore, based on the results of this study, a three-color model is proposed by using the intra-range and interrange of the least uncomfortable postural angles:

- Red, if at least one of the selected three angles exceed its inter-range

- Green, if all the three selected angles are inside their intra-ranges

- Yellow, if at least one of the selected angle exceed the intra-range, but none of them exceed their inter-ranges

Figure 6.

The principal hypothesis of this model is that a 'green' configuration is assumed to have a low level of discomfort, a 'red' with a high discomfort and a yellow the transition between 'green' and 'red'. This is quite similar to the method recently proposed by Naddeo et al (2015) for postural comfort evaluation. The ranges of joint angles in the rest postures (RRP) in the comfortable range of motion (CROM) were defined and considered to be the maximum comfort for the corresponding joints, whereas the extremities of the CROMs represent the minimum 
comfort. Data from Condition 4, after having excluded 15 subjects who had an intra-variability of discomfort rating higher than 10, were used to test if the three-color model is useful for discomfort evaluation. Trunk inclination, left knee angle at the end of clutch pedal travel and left elbow were selected to classify each trial into one of the three colors. The numbers and percentage of each color at different discomfort rating categories are presented in Figure 6. It can be seen that the percentage of green trials decreases and that of red trials increases with discomfort rating as expected. Among 128 trials with a rating higher than 30 (strong or very strong discomfort), only $7 \%$ of them were "green" and $57.8 \%$ were "red". Following suggestions of using the inter- and intra-ranges of comfortable postural angles could be formulated when assessing a vehicle configuration:

- Vehicle interior should be designed approaching to a 'green' configuration so that most of target drivers could adopt the postural variables within their intra ranges for ensuring a low discomfort.

- Modifications should be taken to avoid a 'red' configuration.

- "Yellow" configurations needs to be examined to see if a better compromise could be achieved.

\section{Limitations}

It should be noted that the intra-range boundaries of the least uncomfortable postural angles were estimated by combining the intra-individual variability and the $50^{\text {th }}$ percentile of the distribution of individual medians. Due to large inter individual variability, the intra-ranges proposed in the present study were not intended to be representative of any specific individual, but rather to assess the discomfort of a vehicle configuration.

The selection of trunk inclination, left knee angle at end of the clutch pedal travel and elbow angle as key postural variables for discomfort assessment was the result of an exploratory process. The main raison of this choice is that they were little correlated and other postural angles can be determined if these three angles are known as the position of the feet and hands is highly dependent on the position of the steering wheel and the pedals. However, other alternatives are possible. For example, trunk inclination could be replaced by trunk-thigh angle. Trunk inclination was preferred in this study because it was the least varied angle.

It is believed that the proposed intra and inter ranges of the least uncomfortable angles could be helpful for interpreting predicted postures in terms of discomfort perception and for improving the ergonomics of vehicle interior. Digital human modeling tools (DHM) are largely 
used in automotive industries for assisting in vehicle interior design, in complement with the models directly predicting preferred seat and steering location such as SAE practices. A representative sample of the target population can be generated from an existing anthropometric database (see for instance the method proposed by Parkinson and Reed, 2010); and their postures for a vehicle can be predicted and assessed by the proposed method. However, further investigation and validation on real vehicles are required.

\section{Conclusion}

Both intra and inter individual range of variation of the least uncomfortable driving postural angles were investigated using two different multi-adjustable mock ups. Results show that intraindividual variations of postural angles were much smaller than inter-individual variations as expected. An individual may not feel comfortable throughout the whole range of the least uncomfortable angles from all participants. An exploratory investigation on possible relationship between perceived discomfort and ranges of inter and inter individual variations in least uncomfortable angles suggests that the inter ranges could be used to detect potential problems of postural discomfort while the intra ranges could be considered as optimum ranges. A three-color model, based on the intra-and inter-individual variability ranges of comfortable driving postures, was proposed for ergonomics assessment of a vehicle configuration.

\section{References}

Ausejo, S. and Wang, X., 2009. Motion Capture and human Motions reconstruction. In: Duffy, V.G. (Ed), Handbook of Digital Human Modeling: Research for Applied Ergonomics and Human Factors Engineering. CRC Press, Inc., Boca Raton, FL, USA, 38-1-38-14.

Hiemstra-Van Mastrigt, S., 2015. Comfortable passenger seats: Recommendations for design and research. PhD thesis, TU delft.

Hanson, L., Sperling, L. and Akselsson, R., 2006. Preferred car driving posture using 3-D information. International Journal of Vehicle Design, 42(1), 154-169.

Harrison, D.D., Harrison, S.O., Croft, A.C., Harrison, D.E., and Troyanovich, S.J., 2000. Sitting biomechanics, Part II: Optimal car driver's seat and optimal driver's spinal model. Journal of Manimilative and Physiological Theraoeutics, 23(1), 37-47

Kilincsoy Ü., Wagner A.S. Bengler K., Bubb H., and Vink P., 2014. Comfortable rear seat postures preferred by car passengers. Proceedings of the 5th International Conference on Applied Human Factors and Ergonomics AHFE 2014, Kraków, Poland 19-23 July 2014, Edited by T. Ahram, W. Karwowski and T. Marek

Naddeo A., Cappetti N., and D'Oria C., 2015. Proposal of a new quantitative method for postural comfort evaluation. International Journal of Industrial ergonomics, 48, 25-35. 
Kyung, G., and Maury, A.M., 2009. Specifying comfortable driving postures for ergonomic design and evaluation of the driver workspace using digital human models. Ergonomics, 52, 939-953.

Park, S.J., Kim, C.B., Kim, C.J., and Lee, J.W., 2000. Comfortable driving postures for Koreans. International journal of industrial ergonomics, 26(4), 489-497.

Parkinson, M.B. and Reed, M.P., 2010. Creating virtual user populations by analysis of anthropometric data. International Journal of Industrial Ergonomics, 40, 106-111

Peng, J., Wang, X. and Denninger, L. 2015. Comparing the preferred driving postures between Chinese and French drivers. Proceedings 19th Triennial Congress of the IEA, Melbourne 9-14 August 2015

Porter, J.M.and Gyi D.E., 1998. Exploring the optimum posture for driver comfort. International Journal of Vehicle Design, 19 (3), 255-266.

Rebiffé, R., 1969. Le siège du conducteur :son adaptation aux exigences fonctionnelles et anthropométriques. Ergonomics, 12, 246-261.

Seidl, A., 1994. Das Menschmodell RAMSIS, Analyse, Synthese und Simulation Dreidimensionaler Körperhaltung des Menschen. (The man model RAMSIS: Analysis, Synthesis and Simulation of Threedimensional Human Posture), Technical University of Munich, Germany, Doctoral thesis (in German).

Shen, W. and Parsons, K.C., 1997. Validity and reliability of rating scales for seated pressure discomfort. International Journal of Industrial Ergonomics, 20, 441-461.

Schmidt, S., Amereller, M., Franz, M., Kaiser, R., and Schwirtz, A., 2014. A literature review on optimum and preferred joint angles in automotive sitting posture. Appl Ergon 45, 247-260.

Van der Meulen, P. and Seidl, A., 2007. Ramsis-the leading cad tool for ergonomic analysis of vehicles. Digital Human Modeling. Springer Berlin Heidelberg. 1008-1017.

Vogt, C., Mergl, C., and Bubb, H., 2005. Interior layout design of passenger vehicles with RAMSIS. Human Factors and Ergonomics in Manufacturing \& Service Industries, 15(2), 197-212. 